\title{
The retention of incidentally acquired order information
}

\author{
WILLARD N. RUNQUIST \\ University of Alberta, Edmonton, Alberta, Canada
}

\begin{abstract}
After subjects studied word pairs, they were asked to recall the ordinal position of each pair in the list, both immediately after study and $10 \mathrm{~min}, 48 \mathrm{~h}$, or 1 week later. On the immediate test, they could remember the positions of some of the pairs at the beginning and end of the list, but memory for pairs in the interior of the list was poor. Position information was lost over time. There was no evidence that associative recall depended on the availability of position information, or that the forgetting of item information depended upon the loss of position information.
\end{abstract}

Information in memory that was once retrievable later may be unretrievable for a number of reasons: the cues or other conditions for retrieval may be inadequate, recall of the information may be interfered with by other memories, or the information may have disappeared from memory in some way. The research presented in this paper examines how the conditions of retrieval are involved in forgetting. More specifically, it focuses on contextual attributes, that is, attributes that are not an essential property of the event to be retrieved (Underwood, 1969).

Sometimes context is correlated uniquely with an event at the time it occurs (e.g., the event's temporal position in a series of other discrete events), and sometimes it is not (e.g., the general background environment that may be present when a number of discrete events occur). The general assumption that motivated the experiment presented here is that retrieval may be aided by the presence of these contextual attributes. Some forgetting, then, could occur as a result of the failure of contextual support. If the contextual attributes are themelves memorial in nature, their decreasing availability could produce a decline in the retrievability of particular target events. Thus, if serial position information does support item recall, and its availability decreases over time, the availability of information correlated with it should show a similar and correlated decline.

The direct purpose of this experiment, then, was to investigate the course of forgetting of position information, when this information was acquired incidentally in the encoding of other information. After studying word pairs, presumably for a cued recall test of memory for the words, subjects were asked to produce an estimate of the serial position of various items in the list. These tests were given after intervals of up to 1 week after study in order to as-

This research was supported by a grant from the Natural Sciences and Engineering Research Council of Canada. Louise Renney and Linda Butler supervised the data collection. The author's mailing address is: Department of Psychology, University of Alberta, P-220 Biological Sciences Building, Edmonton, Alberta, Canada T6G 2 E9. sess the long-term retention of any position information acquired.

\section{METHOD}

Each subject studied a single list of 24 pairs of weakly associated words. Two separate lists were used. Each pair was typed on a $3 \times 5$ card with the left-hand member in lowercase and the right-hand member in uppercase. The cards were arranged in decks. On the single study trial, the subject turned a card over and studied the pairs for $5 \mathrm{sec}$ each time a tone was sounded. Subjects were told to memorize the second member of the pair, and they were informed that the word in lowercase would help them remember the word. No indication of the nature of any test was given before study. Immediately following the study trial, subjects worked on a number of mathematical series problems for 2 min.

The next stage in the experiment was a test for serial position on half of the items. The 12 pairs of words were listed on a sheet of paper, and the subject was asked to write the input position of the pair (1-24) next to it. Following this test, subjects were dismissed with instructions to return for a second appointment.

The second session took place $10 \mathrm{~min}, 48 \mathrm{~h}$, or 1 week later. Those subjects who waited $10 \mathrm{~min}$ remained in a waiting room near the laboratory. All others left the laboratory. The first test given each subject at the second session was identical to the test given during the first session except that all 24 pairs were tested. Following this test, all subjects were given a cued recall test. The 24 cue words (lowercase) were printed on a sheet of paper, each followed by a blank space. The subject was given 2 min to write all of the correct associates in the appropriate spaces.

The subjects were introductory psychology students who participated as part of a course laboratory requirement. They were tested in groups of up to 6 students.

The pairs were arranged in six different orders, so that each pair appeared once in each sixth of the list, but within this block, the four pairs always occurred in the same order. There were two sets of items used on the first recall test, with half of the subjects being tested on each set. Two items were selected from each sixth of the list for the first set, and the remaining two comprised the second set. Since there were two entirely separate lists, the balancing procedure required 24 subjects for one replication. The order of the pairs or cue words on the test sheets was the same for all subjects, but was unsystematic with respect to any input order. On the second test, the previously tested items were tested first, but the subject was not informed that this was the case.

\section{RESULTS AND DISCUSSION}

For all analyses, when statistical significance is assessed, the probability of a Type I error was set at .05 . 
The first results to be presented are from the immediate test. In no case was there a significant difference between the various retention interval groups on the immediate test; therefore, these groups have been combined.

Several different measures may be used to index performance on the position estimation task (Zacks, Hasher, Alba, Sanft, \& Rose, 1984). The percentage of correct estimates was determined by considering any estimate within two positions of the actual position as correct. The percentages for each sixth of the list were $40,20,19,18$, 22 , and 38 . Statistically the differences among positions were significant $[\mathrm{F}(5,345)=5.12]$. The mean of the product-moment correlation coefficients between the estimated and actual positions of the items tested for each subject was .48 . Only 43 of the 72 individual correlations were statistically significant.

The third measure is the mean estimated position for items at each position. These means for each sixth of the list were $6.5,11.8,13.8,14.4,14.6$, and $17.6(\mathrm{SD}=$ 9.3). Again, the main effect of serial position was significant $[F(5,345)=64]$. Items that actually occurred later in the list were estimated as having occurred later, but subjects were generally poor at determining the position of items in the interior of the list. In general, then, people do not very effectively encode relative temporal position under associative learning conditions when not explicitly instructed to do so. Even though position effects were statistically significant, an estimate of accountable variance produced $\omega^{2}=.38$.

The delayed retention test produced results on two types of items: those that were tested on the immediate test and those that were not. Results will be presented only for the mean estimated position measure. These data are presented in Table 1 for items in successive sixths of the list. Also included in the table are the values obtained for that particular group on the immediate test. There was little change in mean estimated position after $10 \mathrm{~min}$, but after $48 \mathrm{~h}$ mean estimated position migrated toward the end of the list for items in the first sixth of the list and toward the beginning of the list for items in the last sixth of the list. After a week, these changes were even more noticeable. The effect was generally the same for tested and untested items. The analysis of variance produced two significant effects, an overall effect of serial position

Table 1

Mean Estimated Position for Items in Each Sixth of the List

\begin{tabular}{|c|c|c|c|c|c|c|c|c|c|}
\hline \multirow[b]{3}{*}{ Position } & \multicolumn{9}{|c|}{ Retention Interval } \\
\hline & \multicolumn{3}{|c|}{$10 \mathrm{~min}$} & \multicolumn{3}{|c|}{$48 \mathrm{~h}$} & \multicolumn{3}{|c|}{1 week } \\
\hline & Imm & Test & Untest & Imm & Test & Untest & $\mathrm{Imm}$ & Test & Untest \\
\hline 1 & 6.8 & 7.7 & 6.1 & 6.1 & 8.1 & 9.7 & 6.7 & 8.1 & 8.9 \\
\hline 2 & 11.3 & 12.4 & 12.1 & 12.0 & 12.8 & 12.8 & 12.0 & 11.3 & 12.7 \\
\hline 3 & 13.8 & 14.0 & 13.1 & 13.5 & 13.5 & 11.6 & 14.0 & 11.7 & 12.7 \\
\hline 4 & 13.8 & 12.9 & 13.3 & 14.3 & 13.0 & 11.6 & 15.2 & 13.1 & 12.1 \\
\hline 5 & 14.4 & 13.7 & 13.8 & 13.6 & 13.8 & 11.5 & 15.9 & 12.8 & 14.8 \\
\hline 6 & 17.2 & 17.3 & 15.6 & 17.4 & 15.2 & 13.4 & 18.2 & 12.3 & 12.8 \\
\hline
\end{tabular}

Note-Immediate test: Between subjects, $M S e=165.43$, within subjects, $M S e=85.67$. Retention test: Between subjects, $M S e=77.57$, within subjects, $M S e=93.62$.
Table 2

Percentage of Items from Various Positions Assigned End Positions

\begin{tabular}{cccccc}
\hline & & \multicolumn{4}{c}{ Retention Interval } \\
\cline { 3 - 6 } True Position & Est Position & Imm & $10 \mathrm{~min}$ & $48 \mathrm{~h}$ & 1 week \\
\hline \multirow{2}{*}{1} & 1 & 46 & 49 & 34 & 40 \\
& 6 & 02 & 05 & 09 & 09 \\
6 & 1 & 05 & 05 & 07 & 13 \\
\multirow{2}{*}{$2-5$} & 6 & 42 & 38 & 22 & 14 \\
& 1 & 06 & 11 & 26 & 19 \\
& 6 & 10 & 21 & 19 & 28 \\
\hline
\end{tabular}

$[F(5,759)=29.31]$, and the interaction of position with retention interval $[\mathrm{F}(10,759)=2.20]$. The only remaining factors that produced $F>1.00$ were the main effect of retention interval $[\mathrm{F}(2,69)=1.89, \mathrm{p}=.16]$ and the interaction of testing with retention interval $[\mathrm{F}(2,759)=$ $2.53, \mathrm{p}=.08 \mathrm{]}$. The latter interaction reflected a tendency for position information of untested items to deteriorate somewhat more rapidly than that for test items. The amount of variance accounted for by serial position thus declined over the retention interval. For $10 \mathrm{~min}, 48 \mathrm{~h}$, and 1 week, $\omega^{2}=.35, .16$, and .08 for the tested items' position information, and $.29, .02$, and .10 for the untested items' position information.

The nature of the changes taking place over time is revealed in Table 2, which presents the percentage of items from the ends of the list (Positions 1 and 6) and from the interior of the list (Positions 2-5) that were assigned positions at either end of the list. Tested and untested items have been combined for this analysis, as have the different retention interval groups for the data reported on the immediate test. On the immediate test, slightly over $40 \%$ of the end items were appropriately placed, while less than $20 \%$ of the interior items were placed at either end. The strategy, which has been identified by other researchers (Toglia \& Kimble, 1976), seemed to be that an item not recognized as an end item was assigned unsystematically to a position somewhere in the interior of the list. With the passage of time, there was little change in the correct placement of primacy items, but the proportion of correctly placed terminal items decreased considerably. There was also a clear increase in the number of interior items that were placed at the ends of the list, and again the change was more marked at the terminal end. Whether this reflects less certainty about which items were in the end positions or a change in the "guessing" strategy is not clear.

The results on the final recall test will not be presented. Although these data theoretically could be used to assess the independence of item and position information, the position test exerted profound effects on recall, rendering any conditional analysis impotent.

The results of two other experiments directed to the issue of independence may be briefly noted, however. In the first experiment, the procedure was identical to that of the present study, except that cued recall tests replaced the position naming test. The significant finding was that input position exerted absolutely no effect on recall, or 
on the rate of forgetting. Since the deterioration of position information was position-dependent, this argues for the independence of the two kinds of information. The second experiment also used cued recall tests, but subjects were provided with accurate position information on the second recall test. This information had absolutely no effect on recall performance, or on the rate of forgetting. Thus, there is no evidence from either study that position information is useful in mediating item recall, or that the changes that take place in this information over time bear any relation to forgetting. Indeed, position information in this situation is so poorly articulated that it is unlikely that it could exert any serious effect.

\section{REFERENCES}

Toglia, M. P., \& Kimble, G. A. (1976). Recall and use of serial position information. Journal of Experimental Psychology: Human Learning \& Memory, 2, 431-445.

Underwood, B. J. (1969). Attributes of memory. Psychological Review, 76, 559-573

Zacks, R. T., Hasher, L., Alba, J. W., Sanft, H., \& Rose, K. C. (1984). Is temporal order encoded automatically? Memory \& Cognition, 12, 387-394.

(Manuscript received for publication August 22, 1985.) 